\title{
Target Normal Spin Asymmetry of the Elastic ep-Scattering at Resonance Energy
}

\author{
Dmitry Borisyuk and Alexander Kobushkin * \\ Bogolyubov Institute for Theoretical Physics \\ Metrologicheskaya str. 14-B, 03143, Kiev, Ukraine
}

(Dated: November 19, 2018)

\begin{abstract}
We study the target normal spin asymmetry for the reaction $e p \rightarrow e p$ at electron energy up to few $\mathrm{GeV}$ in the laboratory frame. The asymmetry is proportional to the imaginary part of the reaction scattering amplitude. To estimate the imaginary part of the amplitude we use the unitarity relation and saturate the intermediate hadron states by the proton and resonances from the first, second and third resonance regions. The resonance electromagnetic transition amplitudes, needed to evaluate the asymmetry, are taken from experiment.
\end{abstract}

PACS numbers: 25.30.Bf, 13.88.+e, 14.20.Gk

\section{INTRODUCTION}

A study of the elastic ep-scattering is an important source of information about the internal structure of the proton. Due to the smallness of the fine structure constant, $\alpha \approx 1 / 137$, the first order perturbation term (the one-photon exchange) is assumed to give a main contribution to the electromagnetic transition amplitude. In the one-photon approximation the elastic ep-scattering is described by two quantities, the electric, $G_{E}\left(Q^{2}\right)$, and magnetic, $G_{M}\left(Q^{2}\right)$, form factors.

The form factors $G_{E}$ and $G_{M}$ are usually extracted from cross section data by the Rosenbluth separation method. The database for $G_{E}$ and $G_{M}$ obtained by this method shows that the ratio $G_{E} / G_{M}$ is approximately constant. Recently new precise measurements of the ratio $G_{E} / G_{M}$ were done at Jefferson Lab [1, 2, 3] by the recoil polarization method [4, 5] and yield significantly different results than the Rosenbluth separation method [6].

Because both measurements are based on the one-photon exchange approximation it is natural to assume that this discrepancy may be explained by the second order perturbation term in the $\alpha$ expansion. The $\alpha^{2}$ perturbation term should result in many effects. Its real part contributes to the cross-section and destroys the Rosenbluth formula. The imaginary part appears in one-particle polarization observables of the elastic $e p$-scattering, the target and beam spin asymmetry, which vanish in the one-photon approximation. The present experimental technique makes possible to measure such observables and thus to take under control effects beyond the one-photon approximation.

The aim of the present paper is a calculation of the target spin normal asymmetry in the elastic $e p$-scattering at electron lab. energy $E_{\text {lab }} \lesssim 2 \mathrm{GeV}$.

The imaginary part of the scattering amplitude, which determines the asymmetry, is simply related (through the unitarity condition) to the electroproduction amplitudes of different hadronic states. The so-called "elastic" contribution (i.e. when the hadronic intermediate state, entering the unitarity condition, is the proton) to the asymmetry was calculated in 7]. In [8] authors got strict bounds on the "inelastic" part of the asymmetry using the Schwartz inequality. But, as the authors noted themselves, those bounds highly overestimate the actual values of the asymmetry, especially at high scattering angles.

In the recent work [9] the asymmetry was calculated with $N$ and $\pi N$ intermediate states. Such approach gives a reasonable approximation at low electron energies, but becomes worse as the energy increases.

Contrary to $[9]$, we will calculate the contribution of the resonances in the intermediate state, namely, $P_{33}(1232)$, $D_{13}(1520), S_{11}(1535), F_{15}(1680)$ and $P_{11}(1440)$, using their experimental electroproduction amplitudes. Such approach may be justified at intermediate energy, $E_{\text {lab }} \lesssim 2 \mathrm{GeV}$. For the large momentum transfer region parton model calculations would be more adequate 10 .

It was noted in [9], that the single-spin asymmetry is sensitive to the electroproduction amplitudes in a wide range of photon virtualities, and this may be a new way to access resonance transition form factors. The results of the present work may be useful in planning of such experiments.

The paper in organized as follows. In Section पwe derive general formalism for the asymmetry, in Sections IIIand IV we explain how we describe the resonances and fit their electromagnetic transition amplitudes. Numerical results and conclusions are given Section $\mathrm{D}$

\footnotetext{
*Electronic address: kobushkin@bitp.kiev.ua
} 


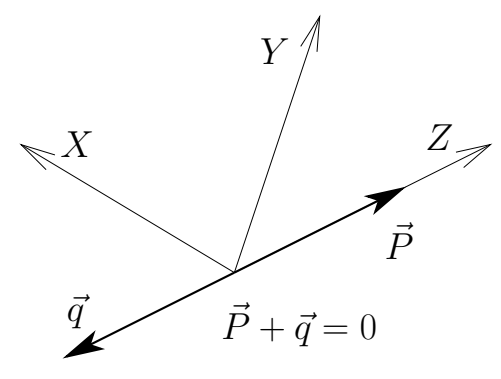

FIG. 1: To the definition of transition amplitudes.

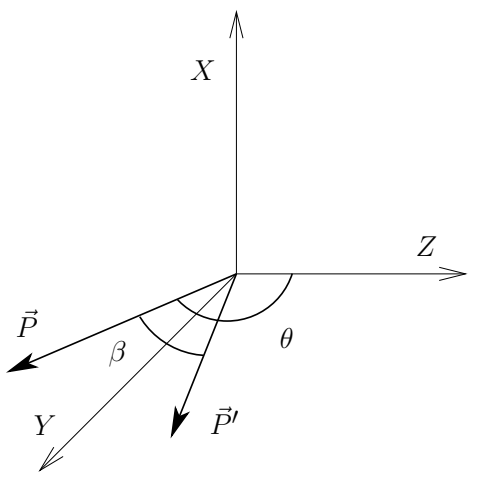

FIG. 2: To the derivation of the hadronic tensor.

\section{GENERAL FORMULAE}

\section{A. Notation}

We denote the initial electron and proton momenta $k$ and $P$, respectively, and the final momenta $k^{\prime}$ and $P^{\prime}$. The transferred momentum is $q=k-k^{\prime}\left(q^{2}<0\right)$, and the c.m. energy squared is $s=(P+k)^{2}=\left(P^{\prime}+k^{\prime}\right)^{2}$. Time and space components of 4-momenta are denoted like $P=\left(\epsilon_{P}, \vec{P}\right) . M$ is the proton mass, the electron mass is neglected. We denote Dirac matrices $\gamma_{\mu}$ and use the short-hand notation $\hat{a}$ for $a_{\mu} \gamma^{\mu}$.

Proton spinors with definite helicity $\lambda$ and momentum $P$ are

$$
u_{\lambda}(P)=\left(\begin{array}{c}
\sqrt{\epsilon_{P}+M} w_{\lambda} \\
\sqrt{\epsilon_{P}-M}(\vec{n} \vec{\sigma}) w_{\lambda}
\end{array}\right) \text {, where } w_{\lambda}=\left(\begin{array}{c}
e^{-i \varphi / 2} \cos \frac{\theta+\pi(1 / 2-\lambda)}{2} \\
e^{i \varphi / 2} \sin \frac{\theta+\pi\left(\frac{1}{2}-\lambda\right)}{2}
\end{array}\right),
$$

where $\theta, \varphi$ are spherical angles of the vector $\vec{n}=\vec{P} /|\vec{P}|, \vec{\sigma}$ are Pauli matrices.

Electromagnetic current matrix elements for the proton read

$$
\left\langle P^{\prime} \lambda^{\prime}\left|J^{\mu}\right| P \lambda\right\rangle=\bar{u}_{\lambda^{\prime}}\left(P^{\prime}\right) \Gamma^{\mu} u_{\lambda}(P)=\bar{u}_{\lambda^{\prime}}\left(P^{\prime}\right)\left[2 M\left(G_{E}-G_{M}\right) \frac{P_{+}^{\mu}}{P_{+}^{2}}+G_{M} \gamma^{\mu}\right] u_{\lambda}(P),
$$

where $|P \lambda\rangle$ is the proton state with momentum $P$ and helicity $\lambda, P_{+}=P+P^{\prime}, G_{E} \equiv G_{E}\left(q^{2}\right)$ and $G_{M} \equiv G_{M}\left(q^{2}\right)$ are the proton elastic form factors.

Current matrix elements between the proton (with momentum $P$ ) and other hadronic states (with momentum $\left.P^{\prime \prime}=P+q\right)$ can be expressed via 3 independent invariant amplitudes. In the rest frame of the hadronic state (see Figure 10 one has

$$
\varepsilon_{\mu}^{(\lambda)}\left\langle h \Lambda\left|J^{\mu}\right| P^{1} / 2\right\rangle=f_{\lambda}^{(h)}\left(q^{2}\right) \delta_{\Lambda, \lambda+1 / 2}, \quad \varepsilon_{\mu}^{(-\lambda)}\left\langle h-\Lambda\left|J^{\mu}\right| P-{ }^{1} / 2\right\rangle=\eta_{h} f_{\lambda}^{(h)}\left(q^{2}\right) \delta_{\Lambda, \lambda+1 / 2} .
$$

Here $\eta_{h}=\pi_{h} e^{i \pi\left(s_{h}-1 / 2\right)}, h$ - some hadronic state, $s_{h}, \pi_{h}$ - its spin and parity, $\Lambda$ - spin projection onto the vector $\vec{P}$. The quantities $f_{\lambda}^{(h)}$ can be considered as helicity amplitudes of the process $\gamma^{*} p \rightarrow h$.

Polarization vectors of a virtual (space-like) photon $\varepsilon_{\mu}$ are defined according to [11. In the coordinate frame of Figure \they are

$$
\varepsilon_{\mu}^{(0)}=\frac{1}{\sqrt{-q^{2}}}\left(|\vec{q}|, 0,0,-q^{0}\right), \quad \varepsilon_{\mu}^{( \pm 1)}=\frac{1}{\sqrt{2}}(0, \mp 1,-i, 0),
$$

where the upper index of $\varepsilon$ shows the spin projection onto the $z$-axis.

If the coordinate system is oriented arbitrarily, so that $\vec{n}=-\vec{q} /|\vec{q}|=(\cos \varphi \sin \theta, \sin \varphi \sin \theta, \cos \theta)$, then

$$
\begin{gathered}
\varepsilon_{\mu}^{(0)}=\frac{1}{\sqrt{-q^{2}}}\left(|\vec{q}|,-q^{0} \vec{n}\right), \\
\varepsilon_{\mu}^{( \pm 1)}=\frac{1}{\sqrt{2}}\{(0, i \sin \varphi,-i \cos \varphi, 0) \mp(0, \cos \varphi \cos \theta, \sin \varphi \cos \theta,-\sin \theta)\} .
\end{gathered}
$$

Orthogonality relations

$$
\sum_{\lambda}(-1)^{\lambda} \varepsilon_{\mu}^{(\lambda)} \stackrel{*}{\varepsilon}_{\nu}^{(\lambda)}=g_{\mu \nu}-\frac{q_{\mu} q_{\nu}}{q^{2}} \quad \text { and } \quad g^{\mu \nu} \varepsilon_{\mu}^{(\lambda)} \stackrel{*}{\varepsilon}_{\nu}^{\left(\lambda^{\prime}\right)}=(-1)^{\lambda} \delta_{\lambda \lambda^{\prime}} .
$$




\section{B. Asymmetry}

The name "target normal asymmetry" corresponds to the situation when the target proton is polarized along the normal to the reaction plane, and other particles are unpolarized. Under such condition the proton spin has two possible directions, say, above and below the reaction plane. Its invariant spin 4-vector should be either collinear or anti-collinear with 4 -vector

$$
S^{\mu}=\frac{2 \varepsilon^{\nu \mu \sigma \tau} k_{\nu} P_{\sigma} P_{\tau}^{\prime}}{\sqrt{q^{2}\left(s q^{2}-\left(s-M^{2}\right)^{2}\right)}}
$$

which is orthogonal to all momenta and satisfies $S^{2}=-1$. The corresponding cross-sections, $\sigma_{\uparrow}$ and $\sigma_{\downarrow}$, are equal in the one-photon approximation, so the difference between them is due to higher order perturbative terms. The target normal asymmetry is defined as dimensionless ratio

$$
A_{n}=\frac{\sigma_{\uparrow}-\sigma_{\downarrow}}{\sigma_{\uparrow}+\sigma_{\downarrow}}
$$

The asymmetry is proportional to the imaginary part of the scattering amplitude. The imaginary part, in turn, can be expressed through the unitarity condition, which reads

$$
i\left(T_{f i}-\stackrel{*}{T} i f\right)=\sum_{n} T_{f n} \stackrel{*}{T}_{i n}
$$

where $i$ and $f$ are initial and final states, respectively, $n$ is so-called intermediate state and $T_{f i}$ are $T$-matrix elements. In our case we can, as the first approximation, use one-photon exchange amplitudes in the right-hand side of (9). Then we obtain

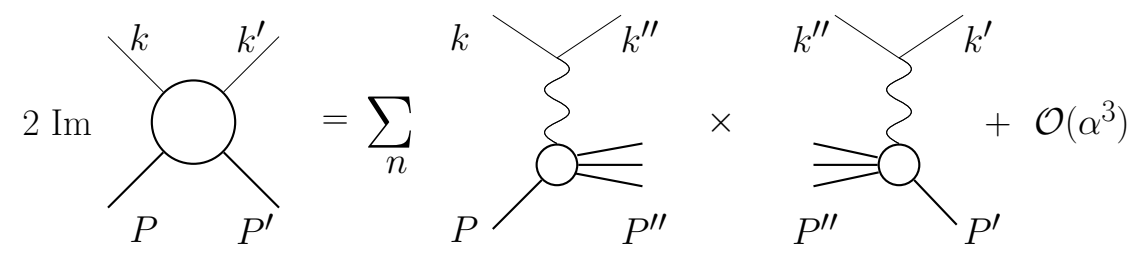

We denote $q_{1}=k-k^{\prime \prime}, q_{2}=k^{\prime}-k^{\prime \prime}$ and the mass of hadronic intermediate state $W=\sqrt{P^{\prime \prime 2}}$.

As it was shown in [8] using (10) and the time-reversal symmetry of the electromagnetic interaction, the asymmetry can be written as [16]

$$
A_{n}=\frac{i \alpha q^{2}}{2 \pi^{2} D} \int_{M^{2}}^{s} \frac{s-W^{2}}{8 s} d W^{2} \int d \Omega_{k^{\prime \prime}} \frac{1}{q_{1}^{2} q_{2}^{2}} L^{\alpha \mu \nu} \sum_{\lambda_{p}, \lambda_{p}^{\prime}} W_{\mu \nu}\left(P^{\prime} \lambda_{p}^{\prime} ; P \lambda_{p}\right) \bar{u}_{\lambda_{p}}(P)\left(-\gamma^{5} \hat{S} \Gamma_{\alpha}\right) u_{\lambda_{p}^{\prime}}\left(P^{\prime}\right)
$$

in the first non-vanishing order of $\alpha$, where $\Gamma_{\alpha}$ is defined in (2),

$$
\begin{gathered}
D=4\left(\frac{\left(2 s+q^{2}-2 M^{2}\right)^{2}}{4 M^{2}-q^{2}}\left(4 M^{2} G_{E}^{2}-q^{2} G_{M}^{2}\right)+q^{2}\left(4 M^{2} G_{E}^{2}+q^{2} G_{M}^{2}\right)\right), \\
L^{\alpha \mu \nu}=\operatorname{Tr}\left(\hat{k}^{\prime} \gamma^{\mu} \hat{k}^{\prime \prime} \gamma^{\nu} \hat{k} \gamma^{\alpha}\right),
\end{gathered}
$$

and the hadronic tensor $W_{\mu \nu}$ is defined as

$$
W_{\mu \nu}\left(P^{\prime} \lambda_{p}^{\prime} ; P \lambda_{p}\right)=\sum_{h}(2 \pi)^{4} \delta\left(P+k-P^{\prime \prime}-k^{\prime \prime}\right)\left\langle P^{\prime} \lambda_{p}^{\prime}\left|J_{\mu}\right| h\right\rangle\left\langle h\left|J_{\nu}\right| P \lambda_{p}\right\rangle .
$$

Here $|h\rangle$ are all possible hadronic states, which we will refer to as "intermediate states". They can be $N, \pi N, \pi \pi N$, $\eta N$ and so on. $\sum_{h}$ is the short-hand notation for

$$
\sum_{N} \sum_{\text {spins }} \int \prod_{a=1}^{N} \frac{d^{3} p_{a}}{(2 \pi)^{3} 2 \epsilon_{p_{a}}}
$$


where $N$ is the total number of particles, $p_{a}$ are their momenta.

Now we will express the hadronic tensor $W_{\mu \nu}$ through the electroproduction helicity amplitudes $f_{\lambda}^{(h)}$.

Consider this tensor in the rest frame of the hadronic state $|h\rangle$, i.e. at $\vec{P}^{\prime \prime}=0$. We choose coordinate system so that both vectors $\vec{P}$ and $\vec{P}^{\prime}$ lie in the $y z$-plane (see Figure 2), and the angle between them is $\beta, 0 \leq \beta \leq \pi$.

The sum $\sum_{h}$ can be split into 2 parts: first, the sum over total angular momentum (=spin) projections, second, the sum over all remaining quantum numbers, which we denote $\sum_{h}^{\prime}$. The behaviour of the state $|h\rangle$ with respect to spatial rotations is completely described by its spin and spin projection, and does not depend on any other quantum numbers. Thanks to this fact, the sum over spin projections can be done explicitly:

$$
\begin{gathered}
W_{\mu \nu}\left(P^{\prime} \lambda_{p}^{\prime} ; P \lambda_{p}\right)=\sum_{h}^{\prime} \sum_{\Lambda^{\prime \prime}}(2 \pi)^{4} \delta\left(P+q_{1}-P^{\prime \prime}\right)\left\langle P^{\prime} \lambda_{p}^{\prime}\left|J_{\mu}\right| h \Lambda^{\prime \prime}\left(\vec{e}_{z}\right)\right\rangle\left\langle h \Lambda^{\prime \prime}\left(\vec{e}_{z}\right)\left|J_{\nu}\right| P \lambda_{p}\right\rangle= \\
=\sum_{h}^{\prime}(2 \pi)^{4} \delta\left(P+q_{1}-P^{\prime \prime}\right) \sum_{\Lambda, \Lambda^{\prime}, \Lambda^{\prime \prime}}\left\langle P^{\prime} \lambda_{p}^{\prime}\left|J_{\mu}\right| n \Lambda^{\prime}\left(\vec{P}^{\prime}\right)\right\rangle\left\langle h \Lambda^{\prime}\left(\vec{P}^{\prime}\right) \mid h \Lambda^{\prime \prime}\left(\vec{e}_{z}\right)\right\rangle\left\langle h \Lambda^{\prime \prime}\left(\vec{e}_{z}\right) \mid h \Lambda(\vec{P})\right\rangle\left\langle h \Lambda(\vec{P})\left|J_{\nu}\right| P \lambda_{p}\right\rangle .
\end{gathered}
$$

Here $|h \Lambda(\vec{a})\rangle$ denotes the state with the spin projection onto vector $\vec{a}$ equal to $\Lambda$.

Wave functions of these states are related via Wigner D-functions [1]]:

$$
\left\langle h \Lambda^{\prime \prime}\left(\vec{e}_{z}\right) \mid h \Lambda(\vec{P})\right\rangle=\mathcal{D}_{\Lambda \Lambda^{\prime \prime}}^{\left(s_{h}\right)}(\varphi, \theta, 0),
$$

where $\varphi, \theta$ are polar angles of the vector $\vec{P}$ and $s_{h}$ is the spin of the state $|h\rangle$. Using (17) and properties of D-functions we have

$$
\left.\sum_{\Lambda^{\prime \prime}}\left\langle h \Lambda^{\prime}\left(\vec{P}^{\prime}\right) \mid h \Lambda^{\prime \prime}\left(\vec{e}_{z}\right)\right\rangle\left\langle h \Lambda^{\prime \prime}\left(\vec{e}_{z}\right) \mid h \Lambda(\vec{P})\right\rangle=\sum_{\Lambda^{\prime \prime}} \mathcal{D}_{\Lambda^{\prime} \Lambda^{\prime \prime}}^{\left(s_{h}\right)}\left(\frac{\pi}{2}, \theta-\beta, 0\right){\stackrel{\mathcal{D}}{\Lambda \Lambda^{\prime \prime}}}_{\left(s_{h}\right)}^{\left(\frac{\pi}{2}\right.}, \theta, 0\right)=\mathcal{D}_{\Lambda \Lambda^{\prime}}^{\left(s_{h}\right)}(0, \beta, 0)
$$

Using also amplitudes definition (3), we obtain

$$
\begin{aligned}
& W_{\mu \nu}\left(P^{\prime} \lambda_{p}^{\prime} ; P \lambda_{p}\right)=\sum_{\lambda, \lambda^{\prime}}(-1)^{\lambda+\lambda^{\prime}} \varepsilon_{1 \nu}^{\left(2 \lambda_{p} \lambda\right)} \stackrel{*}{\varepsilon}_{2 \mu}^{\left(2 \lambda_{p}^{\prime} \lambda^{\prime}\right)} \times
\end{aligned}
$$

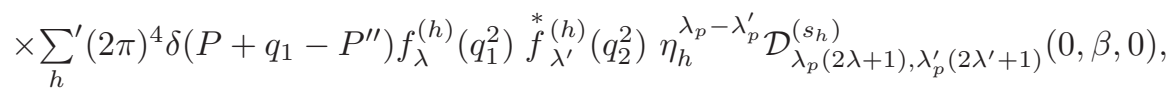

where $\varepsilon_{1}$ and $\varepsilon_{2}$ are polarization vectors of the 1 st $\left(q_{1}\right)$ and 2 nd $\left(q_{2}\right)$ photons of Eq. (10), defined according to (5).

After that the asymmetry becomes

$$
\begin{array}{r}
A_{n}=\frac{\alpha q^{2}}{\pi D} \int_{M^{2}}^{s} \frac{s-W^{2}}{8 s} d W^{2} \int d \Omega_{k^{\prime \prime}} \frac{1}{q_{1}^{2} q_{2}^{2}} \times \\
\times \sum_{h}^{\prime}(2 \pi)^{3} \delta\left(P+k-P^{\prime \prime}-k^{\prime \prime}\right) \sum_{\lambda, \lambda^{\prime}} f_{\lambda}^{(h)}\left(q_{1}^{2}\right) f_{\lambda^{\prime}}^{(h)}\left(q_{2}^{2}\right) X_{\lambda \lambda^{\prime}}^{(h)}\left(W, q_{1}^{2}, q_{2}^{2}\right),
\end{array}
$$

where

$$
\begin{aligned}
X_{\lambda \lambda^{\prime}}^{(h)}\left(W, q_{1}^{2}, q_{2}^{2}\right)=i \sum_{\lambda_{p}, \lambda_{p}^{\prime}}(-1)^{\lambda+\lambda^{\prime}} \eta_{h}^{\lambda_{p}-\lambda_{p}^{\prime}} \mathcal{D}_{\lambda_{p}(2 \lambda+1), \lambda_{p}^{\prime}\left(2 \lambda^{\prime}+1\right)}^{\left(s_{h}\right)}(0, \beta, 0) \times \\
\quad \times L^{\alpha \mu \nu} \varepsilon_{1 \nu}^{\left(2 \lambda_{p} \lambda\right)} \stackrel{*}{\varepsilon}_{2 \mu}^{*\left(2 \lambda_{p}^{\prime} \lambda^{\prime}\right)} \bar{u}_{\lambda_{p}}(P)\left(-\gamma^{5} \hat{S} \Gamma_{\alpha}\right) u_{\lambda_{p}^{\prime}}\left(P^{\prime}\right) .
\end{aligned}
$$

The quantities $X_{\lambda \lambda^{\prime}}^{(h)}$ can be calculated explicitly (provided the proton form factors are known), and the only unknown in (20) are electromagnetic transition amplitudes $f_{\lambda}^{(h)}$.

\section{THE MODEL FOR TRANSITION AMPLITUDES}

Obviously, it is practically impossible to take into account all allowed intermediate states. To proceed further, we need to restrict these states somehow. The authors of [9], for example, included only $N$ and $\pi N$ states. Although 
below the $\pi \pi N$ threshold $\left(E_{\text {lab }} \approx 0.3 \mathrm{GeV}\right)$ such approach gives an exact result, one can expect that as the energy increases, this approximation becomes worse, since more intermediate states (e.g. $\eta N, \pi \pi \pi N$ ) will be possible.

In the present paper we use another way to model the intermediate states. We treat them as a number of resonances and neglect the non-resonant continuum contribution. At present we cannot estimate the non-resonant contribution well enough, but we can give qualitative arguments that it is small.

At a glance one may conclude that the relative size of the non-resonant contribution will be approximately the same as in inelastic cross-sections or structure functions. But actually it is likely to be much smaller for the following reason. Contrary to strictly positive quantities, such as cross-sections, the asymmetry can have either sign. Thus the contributions from different non-resonant states will mostly cancel each other. This is similar to the fact that the average of many uncorrelated random quantities has much smaller dispersion that each of them.

At $P^{\prime}=P$ the hadronic tensor $W_{\mu \nu}\left(P^{\prime} \lambda_{p}^{\prime} ; P \lambda_{p}\right)$, which was introduced in previous section, turns into the hadronic tensor of inelastic ep-scattering. It is natural to assume that the qualitative properties of both tensors are similar, so we should first look what resonances contribute to the inelastic ep-scattering.

There are three prominent resonant peaks in the inelastic ep cross-section: the so-called 1-st, 2-nd and 3-rd resonance regions.

The first resonance peak is due to the $\Delta$-resonance $\left(P_{33}(1232)\right)$, the second peak consists of $D_{13}(1520)$ and $S_{11}(1535)$. There are many resonances which contribute to the third resonance region, but there are serious arguments (see, e.g., Ref. 12]) that the dominant contribution comes from $F_{15}(1680)$. Moreover, it is the only one, for which the transition amplitudes are known. Although the Roper resonance $P_{11}(1440)$ does not contribute significantly to the inelastic $e p$-scattering [12, we also included it in our calculations.

For the proton in the intermediate state one has

$$
\sum_{h}^{\prime}(2 \pi)^{3} \delta\left(P+q_{1}-P^{\prime \prime}\right)=\int \frac{d^{3} P^{\prime \prime}}{2 \epsilon_{P^{\prime \prime}}} \delta\left(P+q_{1}-P^{\prime \prime}\right)=\delta\left(W^{2}-M^{2}\right),
$$

The resonance, however, has a mass $M_{R} \neq M$ and some finite width $\Gamma_{R}$, so we "spread" $\delta$-function with the relativistic Breit-Wigner formula:

$$
\delta\left(W^{2}-M_{R}^{2}\right) \rightarrow \frac{\Gamma_{R} M_{R}}{\pi} \frac{1}{\left(W^{2}-M_{R}^{2}\right)^{2}+M_{R}^{2} \Gamma_{R}^{2}}
$$

After that the expression $\sum_{h}^{\prime}(2 \pi)^{3} \delta\left(P+q-P^{\prime \prime}\right) f^{(h)}\left(q_{1}^{2}\right) f^{*}(h)\left(q_{2}^{2}\right)$, entering the formula for asymmetry, will take the form

$$
f^{(p)}\left(q_{1}^{2}\right) f^{*}(p)\left(q_{2}^{2}\right) \delta\left(W^{2}-M^{2}\right)+\sum_{R} f^{(R)}\left(q_{1}^{2}\right) f^{*}(R)\left(q_{2}^{2}\right) \frac{\Gamma_{R} M_{R}}{\pi} \frac{1}{\left(W^{2}-M_{R}^{2}\right)^{2}+M_{R}^{2} \Gamma_{R}^{2}}
$$

The first part is the "elastic" (proton) contribution; in the second part the sum runs over all resonances taken into account. The quantities $f^{(R)}$ depend only on $q^{2}$ but not $W$. They are related to commonly used [13] $A_{3 / 2}, A_{1 / 2}$, and $S_{1 / 2}$ as

$$
f_{1}=\kappa A_{3 / 2}, \quad \eta_{R} f_{-1}=\kappa A_{1 / 2}, \quad \text { and } \quad f_{0}=\frac{2 q^{2} M_{R}}{\sqrt{4 M^{2} q^{4}-q^{2}\left(M_{R}^{2}-M^{2}-q^{2}\right)^{2}}} \kappa S_{1 / 2}
$$

where $\kappa=\sqrt{\frac{M\left(M_{R}^{2}-M^{2}\right)}{\pi \alpha}}$, while for the proton they are

$$
f_{1}^{(p)}\left(q^{2}\right) \equiv 0, \quad f_{0}^{(p)}\left(q^{2}\right)=2 M G_{E}\left(q^{2}\right), \quad f_{-1}^{(p)}\left(q^{2}\right)=-G_{M}\left(q^{2}\right) \sqrt{-2 q^{2}},
$$

which is easy to derive comparing (2) and (3).

For our calculation we use experimental data on $A_{H}$ (i.e. $A_{3 / 2}, A_{1 / 2}, S_{1 / 2}$ ) given in [13]. Unfortunately, there are no data on $S_{1 / 2}$ for $D_{13}(1520)$ and $F_{15}(1680)$, so in our calculations we set it to zero.

To evaluate the asymmetry we need to fit these data somehow. The fitting procedure is described in the next section. 


\section{FITTING PROCEDURE}

Masses and widths of resonances were taken from PDG 14]. The proton form factors were modelled using the well-known dipole fit

$$
G_{M}\left(q^{2}\right) / \mu_{p}=G_{E}\left(q^{2}\right)=\frac{1}{\left(1+Q^{2} / Q_{0}^{2}\right)^{2}}
$$

where $Q^{2} \equiv-q^{2}, Q_{0}^{2}=0.71 \mathrm{GeV}^{2}$ and $\mu_{p} \approx 2.79$ is the proton magnetic moment.

Now consider the fit of electroproduction amplitudes. According to quark model prediction the high $Q^{2}$ behaviour of the transition amplitudes should be like $A_{1 / 2} \sim S_{1 / 2} \sim Q^{-3}, A_{3 / 2} \sim Q^{-5}[\underline{15}$.

For the proton we have

$$
A_{1 / 2} \sim \sqrt{-q^{2}} G_{M}\left(q^{2}\right) \sim \frac{Q}{\left(1+Q^{2} / Q_{0}^{2}\right)^{2}} \sim Q^{-3} .
$$

The denominator (dipole formula) is entirely due to quark structure, while the numerator is just a kinematical factor. But the fact that $A_{1 / 2}$ tends to zero as $Q^{2} \rightarrow 0$ is the specific feature of the proton. For the other hadronic states $A_{1 / 2}(0) \neq 0$, so like [15] and for the same reasons we introduce factor $\sqrt{\left(M_{R}-M\right)^{2}+Q^{2}}$ and assume, instead of (28),

$$
A_{1 / 2} \sim \frac{\sqrt{\left(M_{R}-M\right)^{2}+Q^{2}}}{\left(1+Q^{2} / Q_{0}^{2}\right)^{2}} .
$$

So, to obtain correct asymptotic behaviour of $A_{1 / 2}$ at $Q^{2} \rightarrow 0$ and $Q^{2} \rightarrow \infty$, it is useful to fit the function

$$
\tilde{A}_{1 / 2}=\frac{\left(1+Q^{2} / Q_{0}^{2}\right)^{2}}{\sqrt{\left(M_{R}-M\right)^{2}+Q^{2}}} A_{1 / 2}
$$

which has finite values at both $Q^{2}=0$ and $Q^{2}=\infty$. The same can be stated for $S_{1 / 2}$. On the other hand, $A_{3 / 2}$ has another asymptotic behaviour $\left(\sim Q^{-5}\right)$, therefore we would have used $\left(1+Q^{2} / Q_{0}^{2}\right)^{3}$ instead of $\left(1+Q^{2} / Q_{0}^{2}\right)^{2}$ in expressions like (29) and (30) for $A_{3 / 2}$.

However the $Q^{2}$ values needed for our calculation are not too high. Since $Q^{2}=\frac{\left(s-M^{2}\right)^{2}}{s} \sin ^{2} \frac{\theta}{2}$, where $\theta$ is the c.m. scattering angle, one gets $Q_{\max }^{2} \sim 3 \mathrm{GeV}^{2}$ at $E_{\text {lab }}=2 \mathrm{GeV}$. Trying different parameterizations, we found that better agreement with the experimental data in the range $Q^{2} \lesssim 3 \mathrm{GeV}^{2}$, especially for Delta, is achieved if we use the same formulae

$$
A_{H} \sim \frac{\sqrt{\left(M_{R}-M\right)^{2}+Q^{2}}}{\left(1+Q^{2} / Q_{0}^{2}\right)^{2}}
$$

for all amplitudes. Thus we fit the functions

$$
\tilde{A}_{H}=\frac{\left(1+Q^{2} / Q_{0}^{2}\right)^{2}}{\sqrt{\left(M_{R}-M\right)^{2}+Q^{2}}} A_{H} .
$$

To describe high $Q^{2}$ behaviour better, we treat $\tilde{A}_{H}$ as a function of $\xi=1-\frac{1}{1+Q^{2} / Q_{0}^{2}}$ instead of $Q^{2}$. At low $Q^{2}$ it does not matter, since $\xi \sim Q^{2}$, but the advantage is that $\xi$ is finite at $Q^{2} \rightarrow \infty$, so we can use simple linear or polynomial fit for all $\xi$ values.

In our calculations we restrict ourselves to the linear least-squares fit of the form

$$
\tilde{A}_{H}(\xi)=a+b \xi
$$

Results of the fit are summarized in Table 1. The corresponding dependence of amplitudes $A_{H}$ vs $Q^{2}$ for all considered resonances is shown on Figure 3 together with experimental points. 


\begin{tabular}{|c|rrr|}
\hline State & \multicolumn{1}{|c}{$\tilde{A}_{3 / 2}$} & $\tilde{A}_{1 / 2}$ & $\tilde{S}_{1 / 2}$ \\
\hline$P_{33}(1232)$ & $-0.929+0.264 \xi$ & $-0.485+0.130 \xi$ & $0.069+0.022 \xi$ \\
$D_{13}(1520)$ & $0.318-0.273 \xi$ & $-0.029-0.474 \xi$ & no data \\
$S_{11}(1535)$ & 0 & $0.123+0.416 \xi$ & $0.212-0.614 \xi$ \\
$F_{15}(1680)$ & $0.185-0.052 \xi$ & $-0.033-0.199 \xi$ & no data \\
$P_{11}(1440)$ & 0 & $-0.351+0.787 \xi$ & $0.236-0.134 \xi$ \\
\hline
\end{tabular}

TABLE I: Fit of the transition amplitudes. All values are in $\mathrm{GeV}^{-1}$.
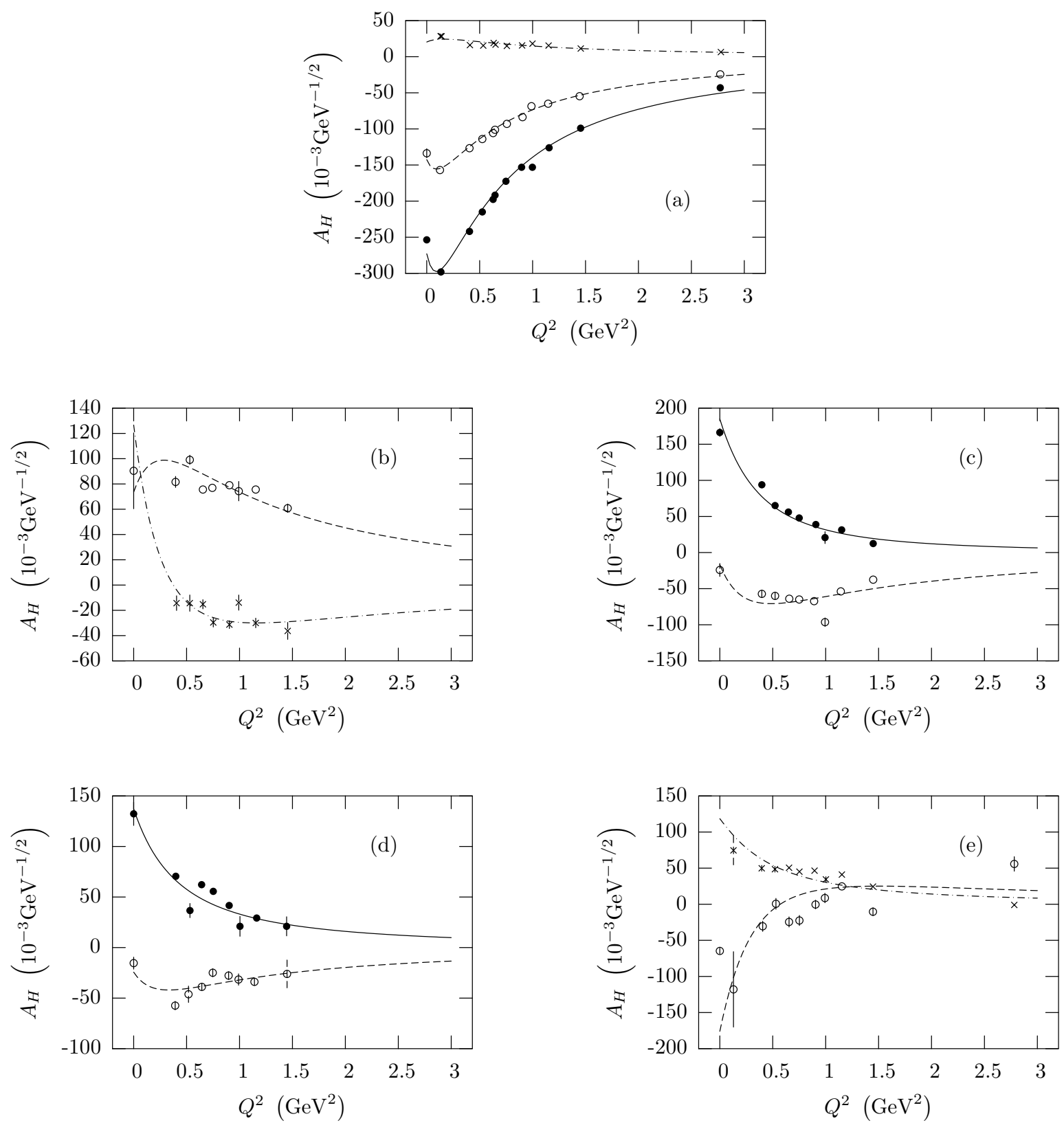

FIG. 3: Fit of the transition amplitudes $A_{3 / 2}$ (solid lines and filled circles), $A_{1 / 2}$ (dashed lines and open circles) and $S_{1 / 2}$ (dash-dotted lines and crosses) for the resonances (a) $P_{33}(1232)$, (b) $S_{11}(1535)$, (c) $D_{13}(1520)$, (d) $F_{15}(1680)$ and (e) $P_{11}(1440)$. Experimental points are from a compilation of [13]. 


\section{NUMERICAL RESULTS AND CONCLUDING REMARKS}

Figure 4 displays the contribution of separate resonances to the target normal asymmetry $A_{n}$ vs the c.m. scattering angle $\theta$ at different electron lab. energy, $E_{\text {lab }}$. One sees that globally the $\Delta(1232)$ contribution is dominant. This is due to its large transition amplitudes, in comparison with other resonances, and the lowest mass among them. The contribution of the Roper resonance was obtained to be not negligible. Moreover at $E_{\text {lab }} \sim 0.9 \mathrm{GeV}$ it becomes comparable with the $\Delta(1232)$ contribution (the upper-right panel of Figure 4). This is very nontrivial fact, because the Roper contribution in inelastic $e N$-scattering is very small. Nevertheless it can be studied in precise measurements of the $A_{n}$ at special kinematical conditions.

One sees also that the contributions from the $\Delta$ and other resonances have mostly opposite sign and tend to cancel each other, especially at high beam energy. It is clearly seen from Figure 5] where we plot the elastic (proton) and inelastic (resonance) parts of the asymmetry and the total asymmetry. The elastic contribution dominates at low energy $\left(E_{\text {lab }}<0.3 \mathrm{GeV}\right)$ and at energy higher than $1.3 \mathrm{GeV}$. It is quite obvious for the low energy, because the energy is insufficient for resonances to be produced. But at high energy it is nontrivial result, which has interesting consequences. As was discussed in Introduction, the asymmetry depends on the imaginary part of the amplitude. But since the real and imaginary parts are connected (via the dispersion relations), we may expect that the real part will also be defined mostly by proton contribution. This is important for the proper interpretation of the proton form factors measurements.

In summary we have calculated the target normal spin asymmetry, $A_{n}$, for the $e^{-} p \rightarrow e^{-} p$ reaction at the electron beam energy up to few $\mathrm{GeV}$ in the laboratory frame. This quantity gives a direct information about the imaginary part of the reaction scattering amplitude and comes from the second and higher order perturbative terms.

To calculate the imaginary part of the amplitude we used unitarity and saturated the intermediate hadron states by the proton (the so-called elastic contribution) and the resonances from the first, second and third resonance regions (the inelastic contribution). We neglect the non-resonant inelastic contribution, which we expect to be small (see section (III). Besides that, the calculated contributions of separate resonances are interesting alone.

Our calculations demonstrate that at special kinematical conditions (the electron lab. energy near 0.9 GeV) the contribution of the Roper resonance $P_{11}(1440)$ becomes comparable with the $\Delta(1232)$-contribution and affects significantly the target asymmetry. It turn, this opens a possibility to study $P_{11}(1440)$ electromagnetic transition amplitudes in precise measurement of the asymmetry.

[1] M.K. Jones et al., Phys. Rev. Lett. 84, 1398 (2000).

[2] O. Gayou et al., Phys. Rev. Lett. 88, 092301 (2002).

[3] V. Punjabi et al., nucl-ex/0501018

[4] A.I. Akhiezer and M.P. Rekalo, Dokl. Akad. Nauk USSR 180, 1081 (1968).

[5] A.I. Akhiezer and M.P. Rekalo, Sov. J. Part. Nucl. 4, 277 (1974).

[6] J. Arrington, Phys. Rev. C68, 034325 (2003); nucl-ex/0305009

[7] A.J.G. Hey, Phys. Rev. D3, 1252-1258 (1971).

[8] A. de Rujula, J.M. Kaplan and E. de Rafael, Nucl. Phys. B35, 365-389 (1971).

[9] B. Pasquini and M. Vanderhaegen, Phys. Rev. C70, 045206 (2004); hep-ph/0405303

[10] Y.C. Chen, A. Afanasev, S.J. Brodsky, C.E. Carlson, M. Vanderhaeghen, Phys.Rev. Lett. 93, 122301 (2004); hep-ph/0403058

[11] V.B. Berestetskii, E.M. Lifshitz, L.P. Pitaevskii. Relativistic quantum theory. Oxford, New York, Pergamon Press, 1971.

[12] P. Stoler, Phys. Rev. D44, 73 (1991).

[13] L. Tiator et al., Eur. Phys. J. A19, 55-60 (2004); nucl-th/0310041

[14] Paticle Data Group, Phys. Lett. B592, 1 (2004).

[15] C.E. Carlson, N.C. Mukhopadhyay, Phys. Rev. Lett. 81, 2646-2649 (1998)

[16] There is a misprint in Ref. [8], a missing factor 8 in the denominator. 

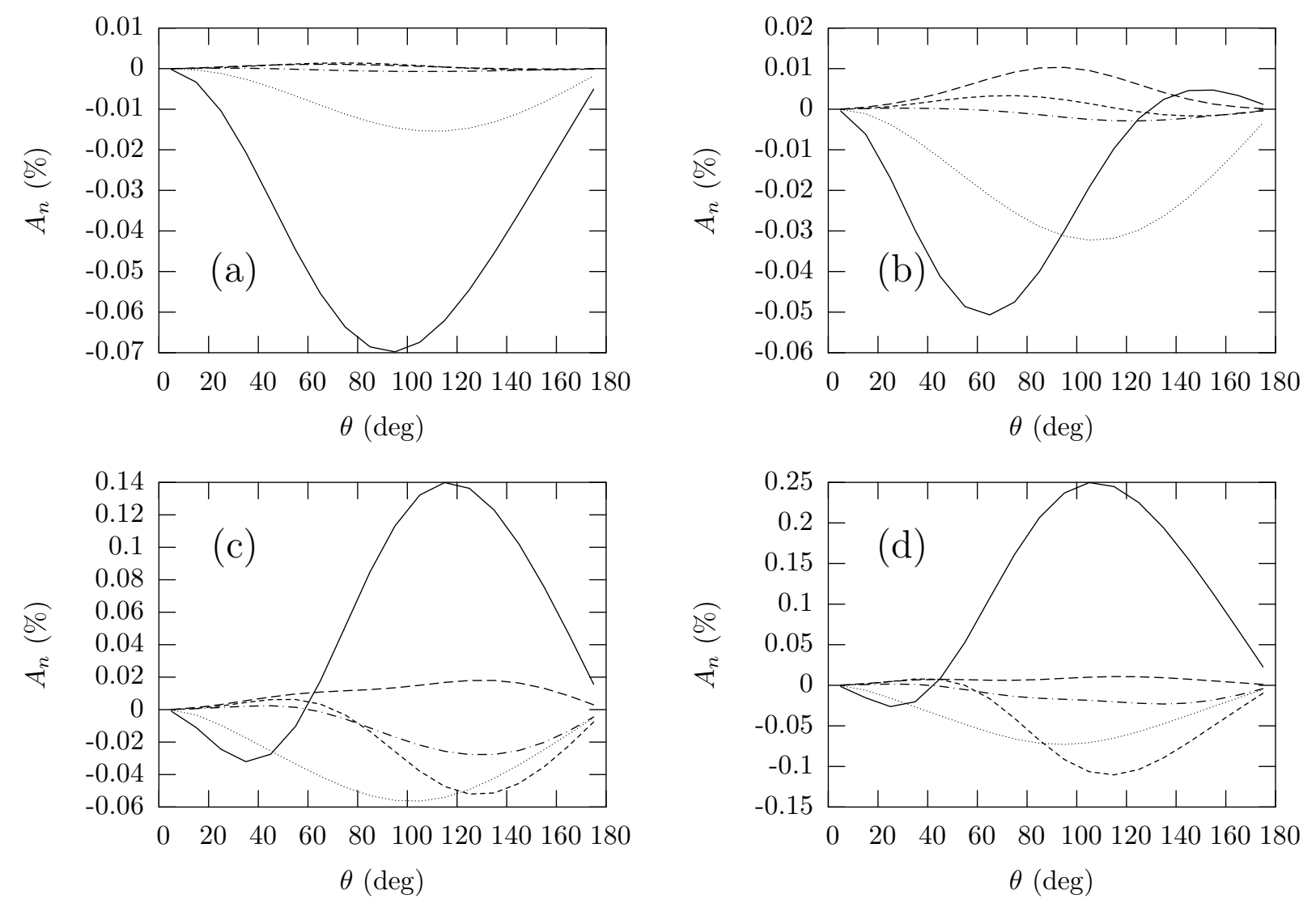

FIG. 4: The contribution of resonances to the asymmetry at different electron lab. energies, (a) $0.57 \mathrm{GeV}$, (b) $0.855 \mathrm{GeV}$, (c) $1.4 \mathrm{GeV}$ and (d) $2 \mathrm{GeV}$. Solid line $-P_{33}(1232)$, long-dashed line $-D_{13}(1520)$, short-dashed line $-S_{11}(1535)$, dash-dotted line $-F_{15}(1680)$ and dotted line $-P_{11}(1440)$.
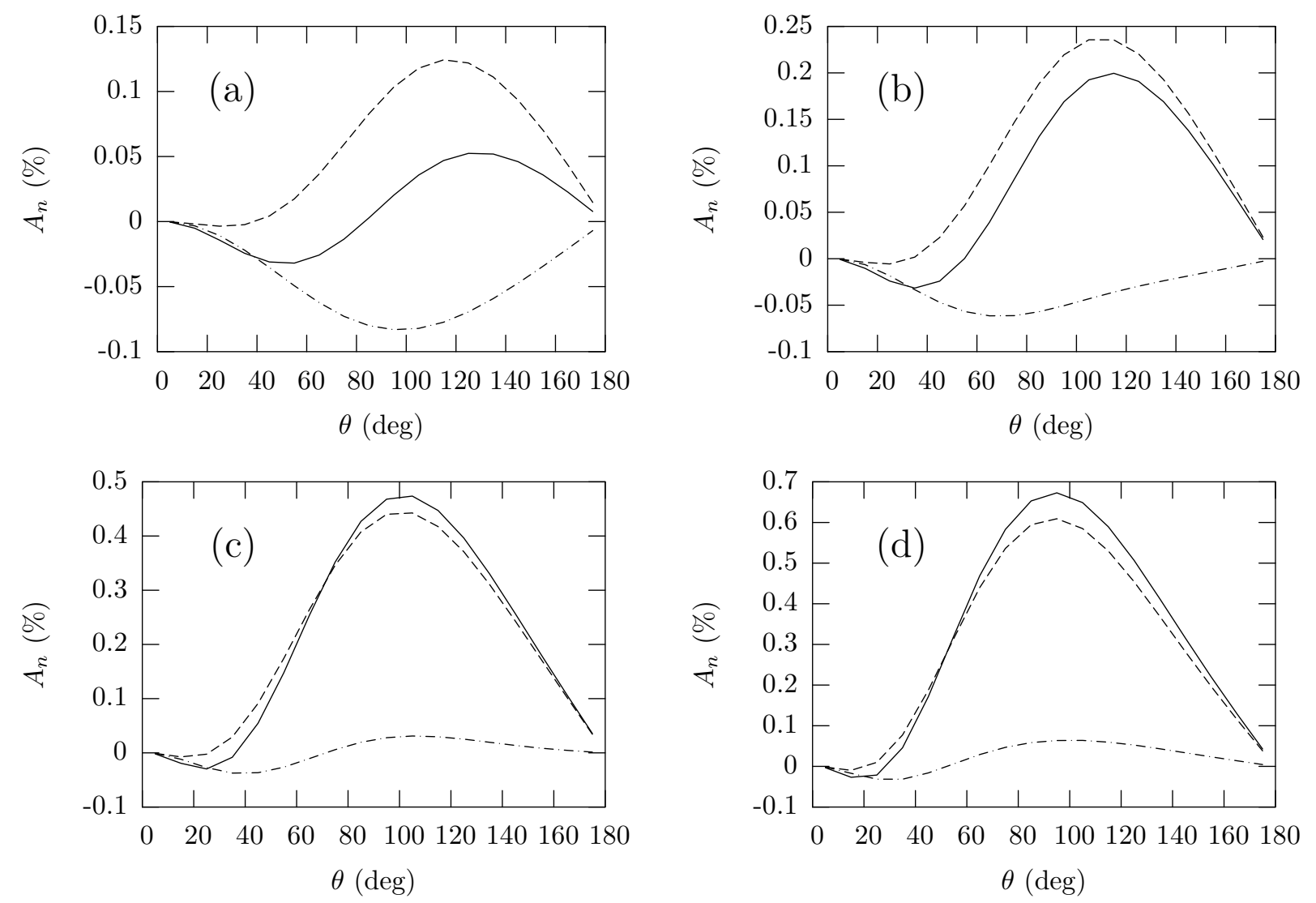

FIG. 5: Target normal spin asymmetry for different electron lab. energies, (a) $0.57 \mathrm{GeV}$, (b) $0.855 \mathrm{GeV}$, (c) $1.4 \mathrm{GeV}$ and (d) $2 \mathrm{GeV}$. The dashed line is the elastic contribution, the dash-dotted line is the inelastic contribution, the solid line is total. 